\title{
Investigation of genetic control of agriculturally important traits in common buckwheat using a combination of genetic mapping and transcriptome analysis
}

\author{
Logacheva M.D. ${ }^{1,2 *}$, Kasianov A.S. ${ }^{1}$, Klepikova A.V. ${ }^{1}$, Makarenko M.S. ${ }^{1}$, \\ Schelkunov M.I. ${ }^{1,2}$, Omelchenko D.O. ${ }^{1}$, Fesenko A.N. ${ }^{3}$, Romanova O.I. ${ }^{4}$, Penin A.A. ${ }^{1}$ \\ ${ }^{1}$ Institute for Information Transmission Problems, Moscow, Russia \\ ${ }^{2}$ Skolkovo Institute of Science and Technology, Moscow, Russia \\ ${ }^{3}$ Federal Scientific Center of Legumes and Groat Crops, Orel, Russia \\ ${ }^{4}$ Federal Research Center Vavilov All-Russian Institute of Plant Genetic Resources, St. Petersburg, \\ Russia \\ * email: maria.log@gmail.com
}

Common buckwheat (Fagopyrum esculentum) is an important non-cereal grain crop. Despite this, little is known about the genetic control of agriculturally important traits in this species. Genetic and genomic studies on buckwheat are hampered by low recombination rate and high abundance of retrotransposons. Here we focused on seed shattering habit, inflorescence structure (determinate vs indeterminate) and seed mass as key traits important for the development of highly productive cultivars. We performed crosses of cultivar Dasha with $F$. esculentum ssp. ancestrale - the wild variety that differs from cultivated ones by small and shattering seeds, highly branched inflorescence, indeterminant growth and prolonged seed dormancy. Using genotypingby-sequencing of 567 F2 plants we reconstructed a high-resolution genetic map of common buckwheat containing 1000000 markers polymorphic between F. esculentum ssp. ancestrale and cultivated varieties. For two traits with monogenic inheritance - seed shattering and inflorescence structure - we identified candidate genes based on sequence similarity and expression profiles using a transcriptome atlas: http://travadb.org/browse/Species=Fesc/. One of the key traits is seed shattering; in order to gain deeper insight into its genetic control we analyzed transcriptomes of pedicels in shattering and non-shattering plants. This allowed to delineate the processes involved in seed shattering; in particular, we found extensive changes in the pathways involved in cell wall modification and cell-to-cell interaction (expansins, catenins, plasmodesmata callose-binding proteins). Seed mass is a highly polygenic trait; genotyping revealed several associated loci though this search is not exhaustive due to the limited number of samples.

Acknowledgements: The study is supported by Russian Science Foundation (project No. 18-76-10008). 